\title{
Organ donation
}

\author{
Nicholas J Odom
}

\section{I-Management of the multiorgan donor}

Shortage of donor organs is the main limiting factor in most types of transplantation. Over 3500 patients are currently awaiting kidney transplants in Britain, and nearly one third of patients accepted for heart transplants die before a suitable donor is found. Potential organ donors are often admitted to peripheral hospitals, where there may be little experience of the issues and problems. These hospitals may each see only one or two such patients a year, so there may be a natural reluctance to get embroiled in the complexities of organ donation.

This article describes the management of the multiorgan donor in the hope that doctors not concerned in transplantation may be encouraged to identify potential donors and to participate in their management.

\section{Identification and assessment of potential donors}

Any patient who is likely to remain permanently unconscious on life support as a result of intracerebral damage should be considered a potential organ donor. This must not influence medical management before brain death is declared, but it does enable subsequent events to proceed more smoothly and rapidly.

The usual causes of brain death are trauma and intracranial haemorrhage. Less common causes are hypoxic brain damage and primary cerebral tumours. Contraindications to organ donation include age ( $>70$ years), malignancy (except primary cerebral tumour), juvenile onset diabetes, intravenous drug abuse, severe multiorgan dysfunction, and systemic infection (minor local infections are not a contraindication).

Doctors caring for potential donors should identify them at such at an early stage and if brain death is considered likely should contact the regional transplant team for further advice. The more warning that transplant teams have the easier it is for them to plan subsequent organ retrieval. This is organised by the regional transplant coordinator, who works with the regional kidney transplant team. The coordinator will

\section{Information needed by transplant team}

- Age, sex, height, approximate weight

- Duration and history of acute illness, including other sites of injury

- Time of intubation

- Condition since admission; periods of hypotension; need for resuscitation

- Previous medical history including previous surgery, alcohol and smoking habits, drug usage, allergies

- Current condition (vital signs, urine output, peripheral perfusion)

- Current medication, including inotropes

- Current investigation results including haemoglobin and white cell count, electrolytes and urea, arterial blood gases, chest radiograph, and electrocardiogram and any positive bacterial cultures. also contact heart and liver transplant teams if the organs appear suitable, either directly or via UK Transplant, the national transplant coordination centre in Bristol.

Activation of organ retrieval procedures can occur only after three conditions have been met: $(a)$ the donor must have been declared brain dead according to the royal colleges' guidelines'; $(b)$ the relatives must have given formal consent to organ donation; $(c)$ if the case is to be referred to the coroner the coroner's officer must have given verbal agreement.

Transplant teams require certain information about the donor, both to assess the suitability of the organs and to select the recipients. Most of this information has already been obtained as part of the assessment of the cerebrally injured patient. Particular items include those given in the box.

Investigations specific for transplantation-Various blood tests are required by transplant teams to facilitate recipient selection and to identify donor transmissible infections. These are organised by the transplant coordinator and include ABO blood group, HLA group, and serological tests for cytomegalovirus, toxoplasma, Epstein-Barr virus, hepatitis, and HIV.

\section{Effects of brain stem death}

Death of the brain stem will inevitably lead to death of all the other organs of the body.' By supporting respiration and other vital functions, however, one can delay deterioration so that other organs may be harvested for transplantation. The aim of supportive care therefore is to keep the other organs in optimum condition for as long as is required for the transplant retrieval teams to arrive and harvest the organs. Furthermore, the better the function of an organ before retrieval, the better the function will be during the first few hours after transplantation. In the case of the heart this may make the difference between life and death.

Consequences of brain stem death on multiorgan function are as follows.

Absence of brain stem reflexes-Respiration is by far the most important brain stem reflex and must be supported by ventilation. The absence of cough reflex means that the airway must be protected and secretions regularly cleared.

Impaired cardiovascular regulation-During the agonal period of brain stem coning there is an intense sympathetic storm, producing severe peripheral vasoconstriction. This may result in left ventricular failure and pulmonary oedema. ${ }^{3}$ This phase is short lived (one to two hours). Established brain stem death causes loss of vascular tone, resulting in hypotension, venous pooling, and relative hypovolaemia. Tachycardia or bradycardia is common. Haemodynamic instability, with changes in vascular tone and cardiac rate, may result from activity within the spinal cord. ${ }^{4}$ 
Impaired thermal regulation-Hyperpyrexia may occur during the initial phase of brain stem injury but is usually short lived. Established brain death results in hypothermia due to metabolic depression and failure to compensate for heat losses.

Deranged hormone metabolism-Diabetes insipidus is the most apparent hormone abnormality. Nevertheless, widespread endocrine disturbances occur after brain death, including depletion of thyroid hormones and depressed adrenocortical function. ${ }^{6}$ These conditions may lead to disruption of mitochondrial function, causing anaerobic metabolism, metabolic acidosis, and eventual cardiac and peripheral circulatory failure.

Spinal reflexes - These may be absent or hyperactive, resulting in exaggerated reactions to painful stimuli and causing hypertension, tachycardia, diaphoresis, and muscle spasms.

\section{General management}

Monitoring-Minimum monitoring requirements are hourly measurements of temperature, pulse, blood pressure, and urine output. Electrocardiographic monitoring is mandatory. Intra-arterial monitoring is helpful since it provides continuous measurement of blood pressure and allows easy access for measurement of blood gases and other tests. A central venous line is not essential provided the donor is in good haemodynamic condition. However, if there are problems in managing haemodynamic and fluid states, or if inotropes are needed, then a central line must be used.

Management of ventilation-Measurement of arterial blood gases is needed for regulating inspired oxygen concentration and minute volume. Ideally oxygen pressure should be above $10 \mathrm{kPa}$ and carbon dioxide pressure around $4.5 \mathrm{kPa}$. Metabolic acidosis should be corrected, but one must be alert to possible causes of acidosis such as low cardiac output and hormonal imbalance, including diabetes mellitus. The endotracheal tube cuff must be airtight to prevent aspiration . Bronchial toilet may be required to clear secretions and must be performed using aseptic technique.

Fluid management-Fluids need to be given intravenously. Although the normal fluid requirement is two to three litres in 24 hours, organ donors may require much more because of diabetes insipidus and other losses, including venous pooling. A good method is to replace the previous hour's urine output with $1 / 5$ isotonic saline in 4\% dextrose, with an additional $500 \mathrm{ml}$ per 24 hours for insensible loss. Other losses should be replaced with appropriate additional fluids - that is, isotonic saline for nasogastric aspirate, and blood or plasma expanders for blood loss or venous pooling. Deviations in plasma sodium concentration can be corrected by appropriate use of normal saline, or $5 \%$ dextrose instead of $1 / 5$ isotonic saline. Potassium losses must also be replaced. Up to $20 \mathrm{mmol}$ potassium or more may be given hourly if plasma concentration is low; this is especially important if there is ventricular arrhythmia. Diabetes insipidus should be treated with desmopressin 1-2 $\mu \mathrm{g}$ intramuscularly, repeated as required. Large doses should not be used, as renal shut down may occur.

Haemodynamic state - The best signs of an adequate cardiac output are a good urine output and a warm periphery. The minimum acceptable urine output is $0.5 \mathrm{ml} / \mathrm{kg} / \mathrm{h}$, although usually the output will be greater than this. A systolic blood pressure of $90 \mathrm{~mm} \mathrm{Hg}$ is satisfactory if peripheral perfusion is good. It is more important to optimise cardiac output and organ perfusion than blood pressure per se. Most donors are in sinus rhythm and many have a tachycardia. Arrhythmias, especially ventricular extrasystoles, may indicate electrolyte imbalance or cardiac contusion.

The commonest cause of inadequate cardiac output is hypovolaemia. Rapid infusion of $250-500 \mathrm{ml}$ of a plasma expander will confirm this diagnosis by producing a fall in pulse rate and an increase in blood pressure and urine output, without a pronounced rise in jugular venous pressure. If these effects are not seen, a central venous line should be inserted. If central venous pressure is low further volume administration should correct the problem; if it is high (more than $8-10 \mathrm{~cm}$ above the mid-axillary line), especially with a low cardiac output, there may be a myocardial metabolic problem or a mechanical problem such as tamponade or contusion.

Inotropic agents should not be used as a substitute for correcting physiological causes of low cardiac output, but when they are required dopamine is the most commonly used, in doses of up to $5 \mu \mathrm{g} / \mathrm{kg} / \mathrm{min}$. Larger doses may sometimes be needed to maintain adequate perfusion of the kidneys and other organs. Sometimes the blood pressure may remain low in spite of a good cardiac output owing to very low peripheral resistance. Under these circumstances systolic pressures below $90 \mathrm{~mm} \mathrm{Hg}$ can be increased using metaraminol $2-5 \mathrm{mg}$ intramuscularly or by slow intravenous infusion. Large doses should be avoided as they cause renal vasoconstriction.

Persistent hypertension can be managed with vasodilators such as hydralazine.

Thermal regulation-Hyperpyrexia can be difficult to treat but normally settles spontaneously. Hypothermia should be managed by close monitoring of core temperature - for example, with a rectal probe-and by reducing heat losses. A core temperature above $35^{\circ} \mathrm{C}$ is a prerequisite for the performance of brain death tests.' A high core temperature with cold periphery is a sign of low cardiac output. Fever may, of course, indicate infection, and any potential donor with fever should have a white cell count and blood cultures. Septicaemia is an absolute contraindication to organ donation.

Prevention of infection - All intravenous lines, urinary catheters, and other tubes should be inserted aseptically. Careful nursing and dressing of wounds is important. The lungs are prone to infection; hence the importance of a well sealed endotracheal cuff, sterile suction catheters, and bagging to prevent atelectasis. Urine, purulent tracheal aspirates, and infected material from other sites should be sent for microscopy and culture. Prophylactic antibiotics should probably be used; the transplant team will advise on this.

Hormone therapy-Intravenous triiodothyronine has been shown to prevent and even reverse many of the metabolic results of brain death, particularly metabolic acidosis, and will diminish or eliminate the need for bicarbonate and inotropic support. ${ }^{78}$ The heart transplant team will advise on dosage. Adrenal suppression can be corrected by giving hydrocortisone $100 \mathrm{mg}$ hourly until the patient is stable and then four hourly. Insulin may be required for hyperglycaemia. There should be no objection to starting hormone therapy as soon as brain death is suspected.

Spinal reflexes-Haemodynamic lability can be reduced by giving narcotic analgesics, which block receptors in the spinal cord. Muscle spasms can be controlled with muscle relaxants; this is especially important during the retrieval operation. These drugs must not be given before brain death tests have been completed.

\section{Individual organs}

Brain-Some procedures designed to protect the brain before brain death has occurred may be harmful to other organs. Hyperventilation, often used to lower intracranial pressure, causes peripheral vasoconstriction and compensatory metabolic acidosis. Restriction 
of fluids to reduce cerebral oedema will diminish cardiac output and renal blood flow.

Kidney-The age limit for donation is around 70, provided the plasma urea and creatinine values were normal on admission and there is no history of renal disease or hypertension. Essential requirements for the kidneys are an adequate cardiac output and fluid input. Diuretics should not be needed unless to treat acute tubular injury due to an episode of hypotension.

Pancreas-Donors under 50 are suitable provided there is no history of diabetes, pancreatitis, or alcohol abuse. ${ }^{y}$ The pancreas is sensitive to ischaemic injury so a good cardiac output is very important.

Liver-Donors under 50 are suitable if there is no history of liver disease, gall stones, or alcohol abuse. ${ }^{10}$ Size matching is important so girth and weight measurements are needed by the transplant team. The liver is sensitive to ischaemic damage so donation is contraindicated if there has been prolonged resuscitation or a systolic blood pressure of less than $70 \mathrm{~mm} \mathrm{Hg}$ for more than 30 minutes. Liver function tests should be performed.

Heart-The age limit is about 50 , but older hearts are more likely to be excluded on inspection because of unexpected atheroma. ${ }^{11}$ Size matching is liberal $( \pm 20 \%$ in body weight under ideal circumstances). The heart should ideally be in sinus rhythm and must be able to sustain good peripheral perfusion and urine output with a low central venous pressure. Subendocardial infarction can occur when there is prolonged external cardiac massage or hypotension (criteria as for liver), and these normally preclude donation. If hypotension has occurred, cardiac enzyme measurement is useful to diagnose subendocardial infarction. Echocardiography may show unsuspected ventricular impairment.

Principles of management are to optimise blood gases, fluids, and electrolytes and to avoid or minimise the use of inotropes, which deplete myocardial high energy phosphates. If dopamine is used for hypotension and the dose cannot be reduced below $5 \mu \mathrm{g} / \mathrm{kg} / \mathrm{min}$ the heart is unlikely to be suitable. Triiodothyronine may reduce inotrope requirements

Lungs-Lungs are normally harvested with the heart for combined transplantation, ${ }^{12}$ though lung transplantation alone is now being done. Heart and lungs from the same donor may therefore be used for different recipients. Donors should be non-smokers under 50 with no history of lung disorder, including asthma. Ventilation for more than three or four days usually makes the lungs unsuitable, as do fractured ribs, pulmonary contusion, pneumothorax, infiltrates on radiography, gross purulent secretions, or pulmonary oedema. Blood gases must be normal at low inspired oxygen concentration, compliance must be high, and lung fields must be completely clear on a chest radiograph, though very mild neurogenic pulmonary oedema may be acceptable. Size matching is critical, so various anthropometric and radiographic measurements are needed. The transplant team will advise on this.
Principles of management are to avoid infection by proper care of the airways and prophylactic antibiotics, maintain as low an inspired oxygen concentration as possible to prevent oxygen toxicity, and avoid overhydration to reduce the likelihood of neurogenic pulmonary oedema. Close monitoring of central venous pressure and urine output are needed to avoid compromising renal function. Volume expansion should be performed only with colloids, and any blood given to the donor should be filtered. The care of potential heart and lung donors is the most demanding task required of the team caring for a multiorgan donor.

Eyes - The only requirements for corneal grafting are that there should be no history of ocular disease and no eye trauma. Eyes can be harvested some hours after cessation of the circulation.

\section{Conclusion}

Care of the multiorgan donor follows basic physiological principles. Transplant teams are always ready to give advice about identification and management of potential donors, and from their point of view good communications and a positive interest on the part of the donor hospital staff are more important than any specific skill.

To increase the number of organs available for transplantation, interest must be encouraged at a local level. Attitudes must change, so that identification and referral of potential organ donors comes to be regarded as a duty rather than an option.

In writing this paper I received advice from Dr S Sterioff, chief of transplantation, and Dr R Steffen, research fellow in liver transplantation, Mayo Clinic, Rochester, USA

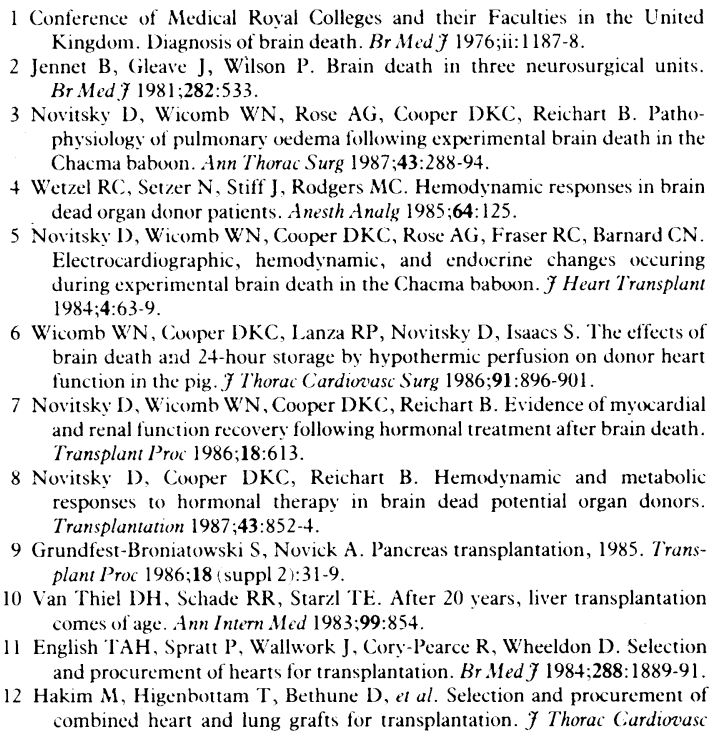

5 Noritsky D, Wicomb WN, Cooper DKC, Rose AG, Fraser RC, Barnard CN Electrocardiographic, hemodynamic, and endocrine changes occuring during experimental brain death in the Chacma baboon. 7 Heart Transplant 1984;4:63-9.

6 Wicomb WN, Cooper DKC, Lanza RP, Novitsky D, Isaacs S. The effects of brain death and 24-hour storage by hypothermic perfusion on donor heart iunction in the pig 7 Thorac Cardiozusc Surg 1986:91:896-901.

7 Novitsk. D) W W W N Cooper DKC Reichart B. Evidence of myocardial . E and renal function recovery foll

8 Noritsk. D, Cooper DKC, Reichart B. Hemodynamic and metabolic responses to hormonal therapy in brain dead potential organ donors. responses to hormonal ther

9 Grundfest-Broniatowski S, Novick A. Pancreas transplantation, 1985. Transplant Proc 1986;18 (suppl 2):31-9.

10 Van Thiel DH, Schade RR, Starzl TE. After 20 vears, liver transplantation comes of age. Ann Intern Med 1983:99:85

11 English TAH, Spratt P, Wallwork J, Cory-Pearce R, Wheeldon D. Selection and procurement of hearts for transplantation. Br.Med 7 1984;288:1889-91

12 Hakim M. Higenbottam T, Bethune D, et al. Selection and procurement of combined heart and lung grafts for transplantation. 7 Thorac Cardiovas Sur $1988: 95 \cdot 47+-9$.

Accepted 9 February 1990
The retrieval of organs for transplantation is usually a complex process. Two or three different transplant teams may take part, often travelling large distances to retrieve organs which need rapid transportation and reimplantation. Organ retrieval is commonly performed in small hospitals where the staff are unfamiliar with the problems and extra demands are made on their often limited time and resources. Such problems may understandably deter staff from referring potential donors.

\section{Donor hospitals}

The primary task of medical and nursing staff in all hospitals is to care for their own patients. It requires some breadth of vision on their part to see that if their patients die they become potential donors for the benefit of other patients they will never meet. Many staff are emotionally affected by the death of a patient and feel reluctant to ask the relatives about organ donation.' 'Caring for a multiorgan donor is demanding and time consuming, and such demands may compete 